\title{
Distribución, abundancia y preferencias alimenticias del pez sapo Opsanus phobetron (Batrachoididae) en la laguna costera de Chelem, Yucatán, México
}

\author{
Walter Gabriel Canto-Maza \& María Eugenia Vega-Cendejas \\ Laboratorio de Taxonomía y Ecología de Peces, CINVESTAV-IPN, Unidad Mérida, km 6 antigua carretera a Progreso, \\ AP 73 Cordemex. 97310 Mérida, Yucatán, México; gcanto@mda.cinvestav.mx
}

Recibido 06-VI-2006. Corregido 15-I-2007. Aceptado 07-V-2007.

\begin{abstract}
Distribution, abundance and alimentary preferences of the fish Opsanus phobetron (Batrachoididae) at the Chelem coastal lagoon, Yucatan, Mexico. Fish are important ecological components in tropical coastal lagoons. In order to evaluate the distribution and feeding preferences of the toadfish Opsanus phobetron in the Chelem Lagoon, Yucatan, Mexico, fish samples were collected using a beach seine in eight stations distributed randomly, from March 2002 to January 2003. The components were analyzed by means of the relative abundance percentage and occurrence frequency. The trophic similarity between ontogenetic stages was determined with the Morisita Index. A total of 221 organisms were collected, with a density and biomass of $92.09 \mathrm{ind} / 100 \mathrm{~m}^{2}$ and $930.39 \mathrm{~g} / 100 \mathrm{~m}^{2}$ respectively. The highest density and biomass were recorded in the same station. A total of 94 stomach contents were analyzed. Results showed a wide trophic generalization, including 40 alimentary items and great ontogenetic variation: juvenile stages consume preferentially microcrustaceans, while adults mainly feed on fish (96 \%). Rev. Biol. Trop. 55 (3-4): 979-988. Epub 2007 December, 28.
\end{abstract}

Key words: Opsanus phobetron, distribution, stomach content analysis, feeding habits, Chelem lagoon. México.

En los sistemas lagunares estuarinos, los peces participan activamente en el proceso de transformación, conducción, intercambio, almacenamiento y regulación de nutrientes (De la Lanza y Canceres 1994). Estos ambientes constituyen importantes rutas de migración en las diferentes etapas del ciclo de vida de varias especies que las utilizan como áreas de crianza, reproducción, refugio y alimentación y representan una disponibilidad de alimento importante debido a la alta productividad que presentan y a la abundancia de recursos alimenticios.

Dentro del componente íctico estuarino, Opsanus phobetron (Walters y Robins 1961) de la familia Batrachoididae, es una especie representativa de la fauna demersal y de importancia en la transferencia de energía en los sistemas costeros de la Península de Yucatán
(Vega-Cendejas 1998). Preferentemente habita en aguas someras sobre sustrato de conchas, con fondos fangosos o arenosos y sobre pastos marinos donde permanecen inmóviles al acecho de su presa. Se caracteriza por ser solitario y de movimiento lento, con una boca grande y oblicua, con fuertes dientes y mandíbulas. Se alimentan principalmente de peces, moluscos y crustáceos.

En el Atlántico occidental se distribuye desde las Bahamas, costas del norte y sur de Cuba, Golfo de México y frente de las costas de Yucatán (Vega-Cendejas et al. 1997). No obstante que esta especie no presenta importancia comercial, su papel ecológico es fundamental en la transformación y trasporte de energía al interior del ecosistema, al constituir recursos alimenticios para especies de niveles tróficos superiores. 
El objetivo de este estudio es describir la distribución, abundancia, biomasa y determinar los componentes que constituyen la alimentación de $O$. phobetron y su variación durante su crecimiento, mediante el análisis de los contenidos estomacales de ejemplares capturados en la laguna de Chelem, Yucatán, México.

\section{MATERIALES Y MÉTODOS}

Área de estudio: la Laguna de Chelem se ubica al noreste de las costas del Estado de Yucatán, $30 \mathrm{Km}$ al norte de la ciudad de Mérida $\left(21^{\circ} 15^{\prime}-21^{\circ} 17^{\prime}\right.$ N y $\left.89^{\circ} 39^{\prime}-89^{\circ} 48^{\prime} \mathrm{W}\right)$. Tiene una superficie aproximada de $15 \mathrm{Km}^{2}$, una longitud y anchura de 14.7 y $1.8 \mathrm{Km}$, respectivamente. Presenta una boca artificial con 225 $\mathrm{m}$ de ancho y una profundidad que varia entre 0.5 y $1.5 \mathrm{~m}$, excepto en la zona de la dársena que alcanza los $3.0 \mathrm{~m}$ (Fig. 1). La temperatura media anual se encuentra entre los 24 y $26^{\circ} \mathrm{C}$. La precipitación media anual es menor a los $500 \mathrm{~mm}$. La evaporación es muy alta presentando sus valores máximos entre abril y junio. La vegetación que la rodea es manglar de bajo desarrollo principalmente Avicennia germinans y Rhizophora mangle, el fondo se encuentra cubierto por Laurencia microcladia, Ruppia maritima y Thalassia testudinum (HerreraSilveira et al. 1998).

Muestreo: los organismos se recolectaron bimensualmente entre marzo, julio, septiembre, noviembre del 2002 y enero del 2003, usando un chinchorro playero de $15 \mathrm{~m}$ de largo por $1.5 \mathrm{~m}$ de caída y apertura de malla de una pulgada, en ocho estaciones distribuidas aleatoriamente (Fig. 1). En cada estación se tomó la temperatura, salinidad y oxígeno disuelto con un multianalizador de campo YSI modelo $85 / 50 \mathrm{FT}( \pm 0.01)$. Las estaciones de muestreo fueron ubicadas geográficamente por medio de un geoposicionador (GPS) digital GARMIN 12XL. Todos los arrastres fueron efectuados entre las 07:00 y las 16:00 h.

Para el análisis de la abundancia, en cada una de las estaciones se determinó la densidad y biomasa como el número de organismos y peso total por unidad de área, extrapolado a 100 $\mathrm{m}^{2}$, así como el peso promedio por individuo (g/ind). Los ejemplares recolectados se preservaron en formaldehído al $30 \%$ y fueron transportados al laboratorio para su identificación, utilizando bibliografía especializada, Guitar (1974), Fischer (1978) y Castro Aguirre et al. (1999). Los juveniles y adultos fueron pesados (peso total) y medidos (longitud estándar). Posteriormente se extrajo el contenido estomacal de cada individuo, y se vació en una caja petri, para ser observado en un microscopio. Los componentes se identificaron hasta el nivel taxonómico más bajo posible según el grado de digestión y con base en bibliografía especializada y se clasificaron en grupos tróficos. Para estimar la variación trófica ontogénica se establecieron clases de longitud de $0.9 \mathrm{~cm}$, obteniendo en total 8 intervalos o clases de tallas. Los componentes en la dieta se identificaron utilizando los siguientes métodos.

Porcentaje por área (PA): es una modificación del método volumétrico indirecto utilizado cuando el contenido estomacal está constituido por presas muy pequeñas (diatomeas, copépodos, ostrácodos) y cuyo peso no es posible registrar en una balanza analítica

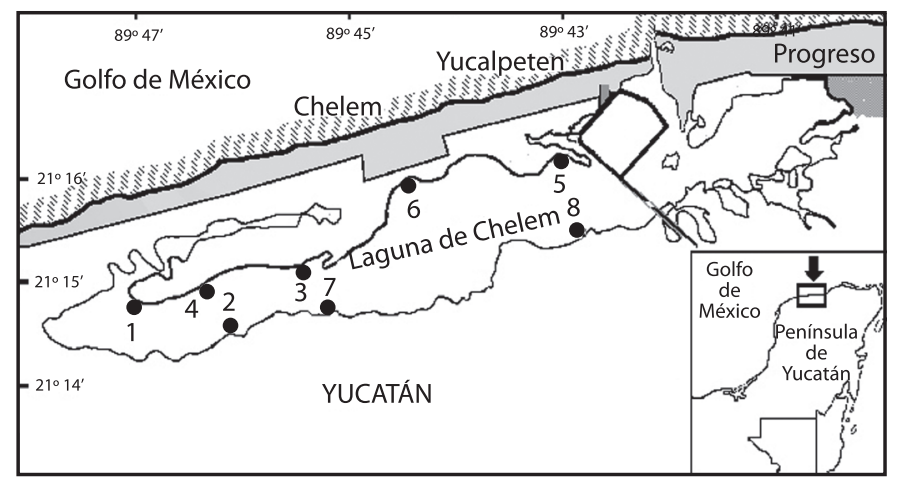

Fig. 1. Área de muestreo y estaciones de colecta de Opsanus phobetron en la Laguna de Chelem Yucatán, México. 
(Vega-Cendejas 1990). Este método proporciona una medida más representativa de la biomasa y puede aplicarse a todos los componentes alimenticios, incluyendo los más pequeños (Hyslop 1980).

Frecuencia de presencia (frequency of ocurrence) (FO): el método de FO consiste en contar el número de veces que aparece una presa " $\mathrm{X}$ " en el contenido estomacal de un grupo de peces y se expresa en porcentaje $(\% \mathrm{FO}=(\mathrm{n} /$ NE) (100)) (Tresierra y Culquichicóm 1993). Este método fue utilizado como complemento del PA para precisar si un componente dado es accidental o es el alimento preferencial en la dieta del depredador (Yáñez-Arancibia 1976).

Se utilizó un análisis de conglomerados por medio del índice de Morisita (1959) modificado por Horn (1966), como ayuda para poder describir los cambios en la composición del alimento con el crecimiento de talla del depredador. Este índice es independiente del tamaño de la muestra y de la diversidad de las presas dentro de la muestra (Wolda 1981).

\section{RESULTADOS}

Variables hidrológicas: el mayor promedio de temperatura y salinidad se registró en la época de lluvias. En el mes de julio se registró $31.2{ }^{\circ} \mathrm{C}$ y en el mes de septiembre una salinidad de $41.0 \%$ o (Cuadro 1). Espacialmente la temperatura más alta se registró en la estación seis con $29.5^{\circ} \mathrm{C}$ y la menor en la estación uno con $25.22{ }^{\circ} \mathrm{C}$. En cuanto a la salinidad el mayor promedio se obtuvo en la estación uno con $39.42 \%$ y la menor para la estación tres con $32.88 \%$ o (Fig. 2).

Densidad y abundancia: se capturaron un total de 221 ejemplares con un peso total de $2233.58 \mathrm{~g}$, los rangos de talla y peso de los organismos capturados fueron entre 1.9 y 15.4 $\mathrm{cm}$ de longitud estándar (LE) y 0.21-62.8 g respectivamente. Se obtuvo una densidad total de $92.09 \mathrm{ind} / 100 \mathrm{~m}^{2}$ y una biomasa total de $930.39 \mathrm{~g} / 100 \mathrm{~m}^{2}$. La mayor densidad y biomasa se registró en el mes de noviembre el cual pertenece a la época de nortes, con una densidad

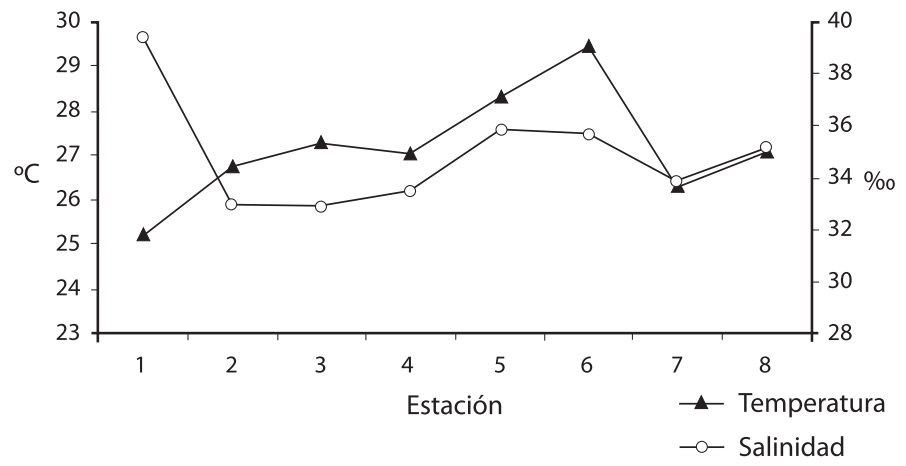

Fig. 2. Valores promedio de la temperatura y salinidad por estación, marzo 2002enero 2003, en la Laguna de Chelem Yucatán, México.

\section{CUADRO 1}

Valores medios de los parámetros hidrológicos ( \pm Desviación estándar) por mes para la laguna de Chelem en el periodo de marzo 2002-enero 2003
Secas
Lluvias
Nortes

$\begin{array}{lccccc}\text { Parámetros } & \text { marzo-02 } & \text { julio-02 } & \text { septiembre-02 } & \text { noviembre-02 } & \text { enero-03 } \\ \text { Temperatura }\left({ }^{\circ} \mathrm{C}\right) & 29.8(1.7) & 31.2(1.5) & 31.0(1.4) & 22.9(1.9) & 21.7(2.4) \\ \text { Salinidad }(\%) & 36.8(1.3) & 38.9(1.5) & 41.0(8.3) & 29.0(2.6) & 29.3(4.6) \\ \text { Oxígeno }\left(\mathrm{O}^{2} \mathrm{mg} / \mathrm{l}\right) & 5.8(2.2) & 9.8(3.8) & 8.7(2.3) & 14.1(3.0) & 15.2(1.9) \\ \text { Profundidad }(\mathrm{m}) & 0.57(0.05) & 0.57(0.12) & 0.74(0.20) & 0.55(0.08) & 0.47(0.07)\end{array}$


de $50.40 \mathrm{ind} / 100 \mathrm{~m}^{2} \mathrm{y}$ una biomasa de 368.98 $\mathrm{g} / 100 \mathrm{~m}^{2}$. El único mes en el cual no se capturó ningún ejemplar fue en marzo (época de secas) (Cuadro 2). En la Fig. 3 se observa el comportamiento de la abundancia de $O$. phobetron en cada una de las estaciones de colecta durante los meses de muestreo. Destaca la estación cuatro por presentar los valores más altos en densidad (26.66 ind $\left./ 100 \mathrm{~m}^{2}\right)$ y biomasa $(254.58$ $\left.\mathrm{g} / 100 \mathrm{~m}^{2}\right)$, y en cuanto al peso promedio la estación seis y siete (17.49 $\mathrm{g} /$ ind y $18.47 \mathrm{~g} /$ ind) presentan los valores mas altos. De los ejemplares capturados el mayor porcentaje estuvo comprendido para individuos con intervalos de talla de 3-10.9 $\mathrm{cm}$ de longitud estándar, los ejemplares mayores de $11 \mathrm{~cm}$ de LE presentaron los valores más bajos durante el periodo de estudio (Fig. 4).

Biología trófica: se analizaron un total de 94 de estómagos de $O$. phobetron con intervalo de tallas entre $2.1 \mathrm{y}$ $13.0 \mathrm{~cm}$ de LE colectados en los meses de julio, septiembre y noviembre. Del total de los estómagos analizados el 9.6 $\%$ estaban llenos, el $37.2 \%$ se encontraban con algo de alimento y el $53.2 \%$ se hallaban casi vacíos.
Composición de la dieta: A partir de los resultados se determinó que esta especie presenta polifagia, incluyendo 40 componentes alimenticios. Los taxones identificados se agrupan en 12 grupos tróficos, dentro de los componentes alimenticios se registraron foramíniferos, anélidos, moluscos, diversos grupos de microcrustáceos y macrocrustáceos, así como equinodermos y peces (Cuadro 3). Respecto a la abundancia (PA), el grupo de
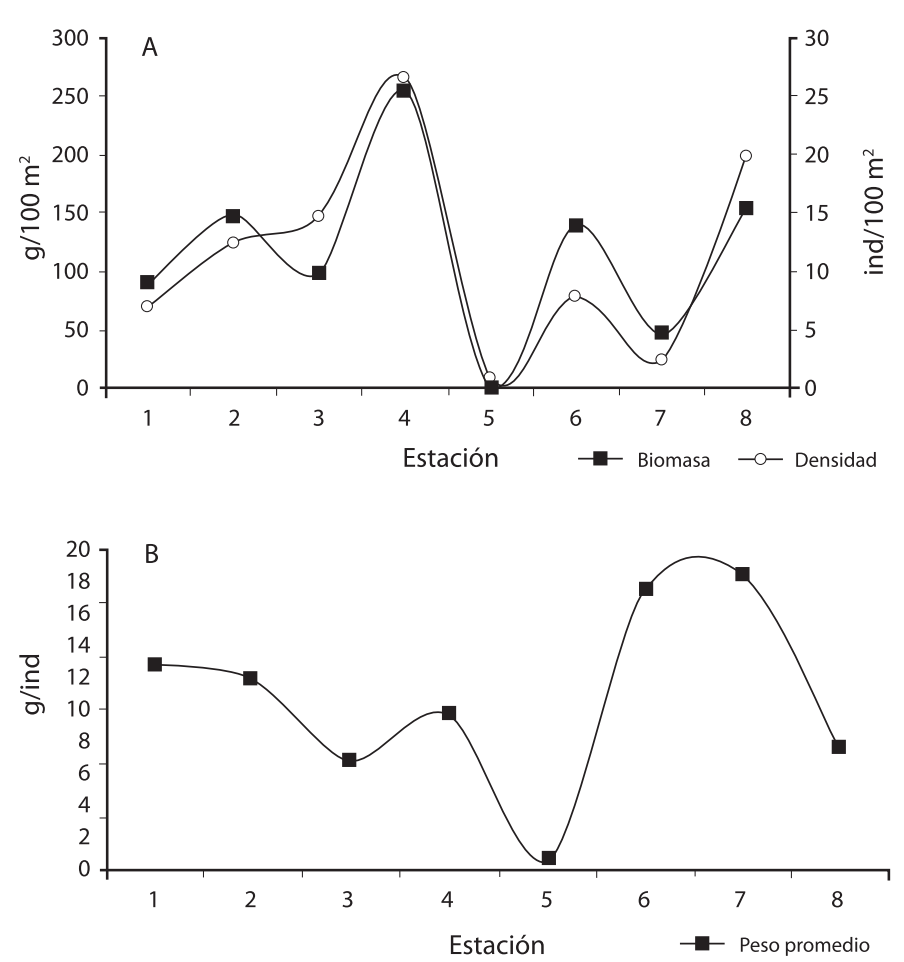

Fig.3. (A) Variación espacial en la densidad y biomasa; y (B) Peso promedio por individuo de Opsanus phobetron en la Laguna de Chelem, Yucatán, México.

CUADRO 2

Densidad (ind/100 $\mathrm{m}^{2}$ y Biomasa $\left(\mathrm{g} / 100 \mathrm{~m}^{2}\right)$ del promedio mensual de Opsanus phobetron recolectada en la laguna de Chelem en el periodo marzo 2002-enero 2003

\begin{tabular}{|c|c|c|c|c|c|}
\hline & Secas & & & & \\
\hline & marzo-02 & julio-02 & septiembre- 02 & noviembre-02 & enero-03 \\
\hline Densidad & 0.00 & 24.16 & 16.66 & 50.40 & 0.83 \\
\hline Biomasa & 0.00 & 315.75 & 215.79 & 368.98 & 29.87 \\
\hline
\end{tabular}




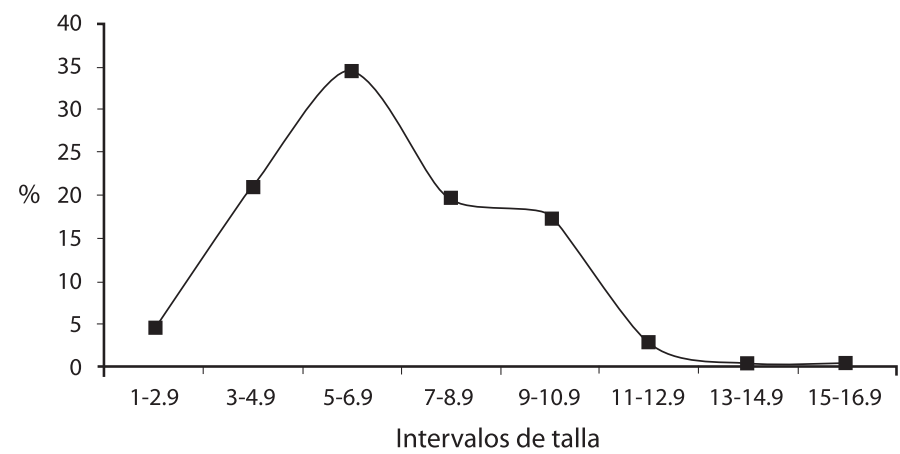

Fig. 4. Intervalo de tallas de los ejemplares de Opsanus phobetron colectados en la Laguna de Chelem, Yucatán, México.

los microcrustáceos es el más representativo (33.10\%), siguiéndole en importancia los peces (18.98), gasterópodos (18.93\%), macrocrustáceos $(10.57 \%)$ y MANI $(8.33 \%)$.

Dentro de los recursos alimenticios registrados, los microcrustráceos fueron el grupo más representativo, siguiéndole en importancia los peces, gasterópodos, macrocrustáceos, restos de MANI (se consideró como MANI a la materia animal no identificada por su grado de digestión.) y dentro de la categoría de "otros" se incluyeron taxas cuya representatividad es baja (Cuadro 3). Respecto a su presencia, sobresalen con una frecuencia mayor del $20 \%$ diversos grupos alimenticios pertenecientes a los microcrustáceos como los isópodos (familias Cirolanidae y Sphaeromidae) y anfípodos, MANI, restos de peces, pastos marinos y los peneidos. Los componentes alimenticios con FO inferiores al $10 \%$ se consideraron como incidentales (Cuadro 3). A nivel de grandes grupos, los micrucrustáceos y los moluscos constituyeron los recursos alimenticios que se registraron en el $99 \%$ y $57 \%$ de los contenidos alimenticios analizados.

Variación trófica ontogenética: en ejemplares juveniles de talla de 2.1 a $4.0 \mathrm{~cm}$ de LE su preferencia alimenticia son los microcrustáceos con el $72 \%$. En los intervalos de talla de 4.1 a $8.0 \mathrm{~cm}$ de LE aumenta el consumo por los peces (23\%), gasterópodos (17\%) y macrocrustáceos (12\%), sin embargo, los microcrustáceos siguen siendo el ítem alimenticio preferencial con el
$38 \%$. Conforme alcanza tallas mayores de 8.1 a $12.0 \mathrm{~cm}$ de LE se observa que los peces y macrocrustáceos siguen formando parte importante en su dieta con el 15 y $9 \%$ respectivamente, sin embargo, se aprecia un aumento considerable en el consumo de gasterópodos y macrófitas, contribuyendo con el $47 \%$ del contenido estomacal. En los ejemplares de 12.0 a $13.0 \mathrm{~cm}$ de LE el grupo alimenticio preferencial son los peces con el $96 \%$ (Fig. 5).

De acuerdo al índice de similitud de Morisita las proporciones de los ítems alimenticios consumidos por esta especie varían de acuerdo con la talla del pez. El análisis con un nivel de corte de 0.25 resalta esta variación en la que se observa la formación de cuatro etapas tróficas con una diversidad trófica característica (Fig. 6). En la primera etapa consumen microcrustáceos y macrocrustáceos. En la segunda etapa consume peces, microcrustáceos, gasterópodos y macrocrustáceos. En la cuarta etapa consume macrocrustáceos, microcrustáceos y macrófitas. En la quinta etapa los gasterópodos y los peces son el principal grupo. En las cuatro etapas existe una similitud en el consumo de microcrustáceos, gasterópodos y peces.

\section{DISCUSIÓN}

Las características hidrológicas dentro de la Laguna de Chelem están asociadas a la morfología y tamaño del sistema, a la influencia de las mareas, al aporte de agua dulce por manantiales, a las diferentes condiciones climáticas, la evaporación, a sí como por factores internos como la actividad biológica. Durante el período de estudio (2002-2003), la temperatura registrada en el sistema fue muy homogénea espacial y temporalmente, con los valores máximos registrados en la época de lluvias y los mínimos en enero debido a la influencia de los nortes. Respecto a la salinidad, espacialmente es muy 
CUADRO 3

Composición porcentual (PA) y frecuencia de presencia (F.O.) en \% de los artículos alimenticios consumidos por Opsanus phobetron $(N=94)$, en la Laguna de Chelem, Yucatán

Artículo alimentario

$\mathrm{PA}(\%) \quad$ FO $(\%)$

Artículo alimentario

PA (\%)

FO (\%)

\section{PROTOZOA}

Foraminiferida

Globigerina sp.

Spiroculina sp.

Triloculina sp.

Total

\section{MACRÓFITAS}

Pasto no ident.

Algas no ident.

Semillas de algas

Total

$\begin{array}{ll}0.04 & 1.06 \\ 0.06 & 2.13 \\ 0.18 & 0.13 \\ 0.28 & 3.32\end{array}$

\section{ANNELIDAE}

Polychaeta

Total

\section{MOLLUSCA}

Bivalvia

Bivalvia

Restos de bivalvo

Total

\subsection{5}

1.50

0.16

6.81

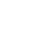

0.46

0.46

2.13

2.13

22.34

2.13

2.13

26.6

Gasteropoda

Bullidae

Caecidae

Cerithiidae

Crepidulidae

Haminoeeidae

Littorinidae

Marginellidae

Modulidae

Veneridae

Phasianellidae

Total

0.12

0.65

0.77

4.67

0.74

1.27

0.18

0.36

0.30

7.35

2.96

0.98

0.12

18.93

\section{MICROCRUSTÁCEOS}

\section{Amphipoda}

7.88

Isopoda

Cirolanidae

Sphaeromidae

Ostracoda

Tanaidacea

Total

\section{MACROCRUSTÁCEOS \\ Brachyura

$$
\text { Portunidae }
$$

1.16

3.19

Decapoda

Caridea

Penaeidae

1.61

7.80

10.57

9.57

Total

21.28

34.04

\section{ECHINODERMATA}

$\begin{array}{lll}\text { Ophiuroidea } & 0.32 & 1.06 \\ \text { Total } & 0.32 & 1.06\end{array}$

\section{PECES}

$\begin{array}{lcc}\text { Lucania parva } & 1.11 & 3.19 \\ \text { Peces no ident. } & 17.87 & 35.11 \\ \text { Total } & 18.98 & 38.30\end{array}$

\section{DETRITUS}

\section{Detritus}

0.33

2.13

Total

0.33

2.13

MANI

Mani

8.33

36.17

Total

OTROS

Amphora sp. $\quad 0.03 \quad 2.13$

Navicula sp. $\quad 0.03 \quad 1.06$

Peridinium sp. $\quad 0.04 \quad 1.06$

Hemiptera $\quad 0.20 \quad 1.06$

Huevo no ident. $\quad 0.79 \quad 1.06$

Nemátodo no ident. $\quad 0.03 \quad 1.06$

$\begin{array}{lll}\text { Total } & 1.12 & 7.43\end{array}$




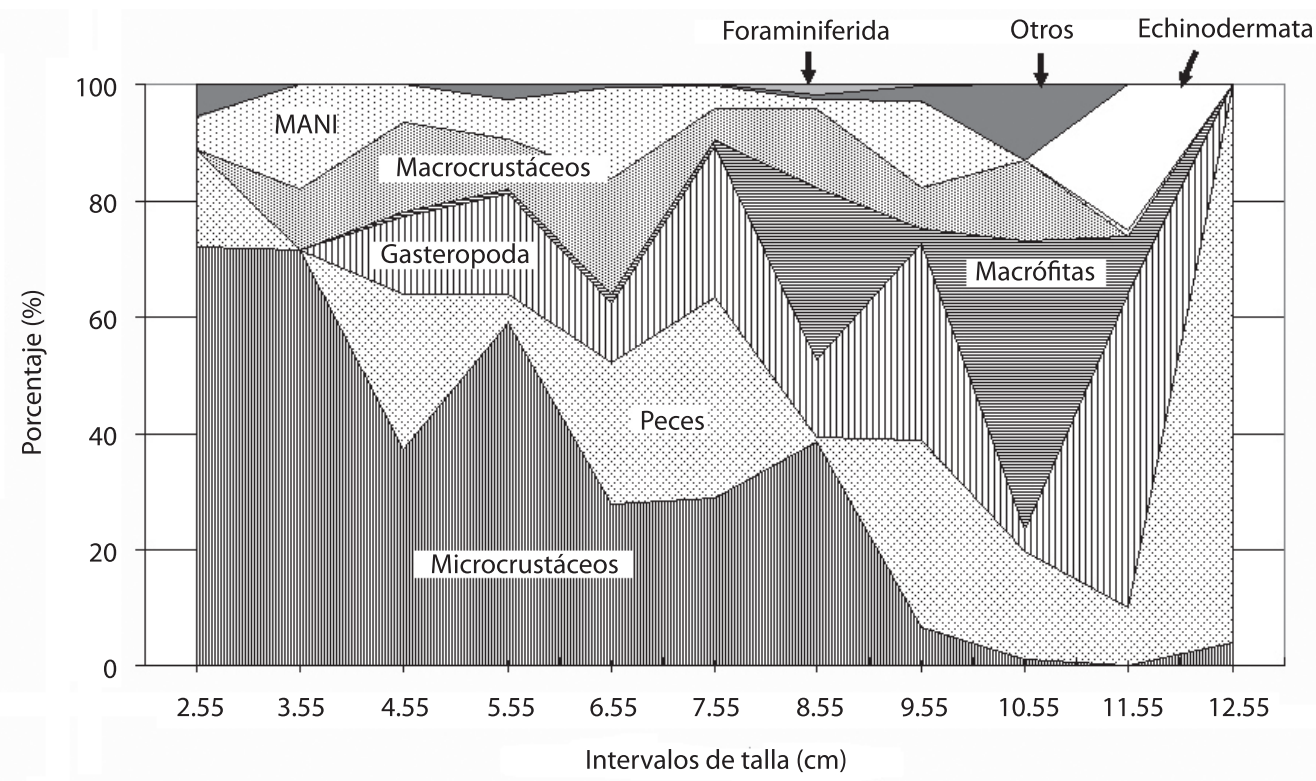

Fig. 5. Variación del espectro trófico entre diferentes intervalos de talla de Opsanus phobetron, de julio, septiembre y noviembre, en la Laguna de Chelem Yucatán, México.

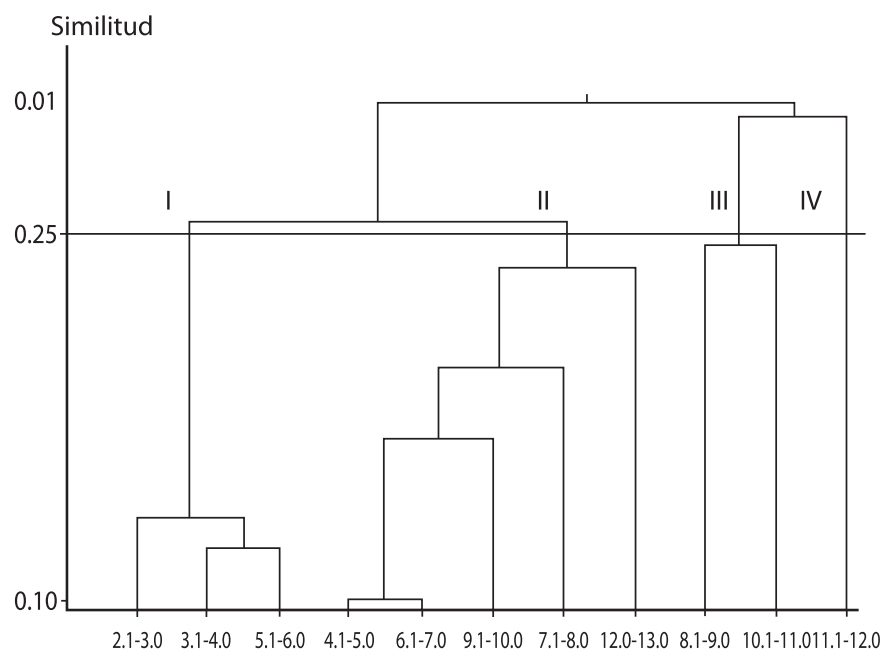

Fig. 6. Dendrograma de similitud trófica a partir del índice de Morisita (1959) que representa a las diferentes unidades tróficas durante la ontogenia de Opsanus phobetron de julio, septiembre y noviembre, en la Laguna de Chelem Yucatán, México.

uniforme, sobresaliendo la estación ubicada en la zona interna (No. 1) con el promedio más alto $(39.42 \%$ ) debido a la poca profundidad. Estacionalmente, la salinidad está influida por el aporte de las descargas fluviales y la influencia de las mareas, con los valores máximos en la época de secas y lluvias y los mínimos en nortes debido al mayor influjo de corrientes de marea.

La presencia y abundancia estacional de $O$. phobetron está relacionada con las características hidrológicas del sistema. Walters y Robins (1961) indican que el límite de distribución de esta especie está relacionado con la temperatura, requiriendo temperatura entre $19-20{ }^{\circ} \mathrm{C}$ para iniciar el desove. Respecto al oxígeno, en el mes de marzo (secas), es cuando se registran los valores más bajos de oxígeno disuelto $(5.8 \mathrm{mg} / \mathrm{l}) \mathrm{y}$ alta salinidad (37\%), no se registró está especie en el sistema lagunar. Sin embargo, en enero cuando la salinidad decrece (29\%) y los niveles de oxígeno se incrementan $(14.1 \mathrm{mg} / \mathrm{l})$ debido a la influencia del oleaje y movimiento de las mareas, se determinaron los valores más altos de abundancia numérica 
y por peso. La presencia de gran número de individuos de esta especie durante esta época del año, sugiere que la laguna de Chelem es utilizada como zona de protección durante este período de mayor influencia de los nortes (Vargas 2004). Estos mismos resultados han sido reportados por Arceo (2005) en donde reporta la mayor abundancia de esta especie en la época de nortes en laguna de Dzilam, Yucatán.

Los análisis de los contenidos estomacales mostraron que $O$. phobetron presenta una alta generalización trófica al incluir en su alimentación diversas presas, así como un consumo preferencial a nivel de grandes grupos, de los microcrustáceos y moluscos, los cuales se encontraron en más del $50 \%$ de los ejemplares analizados. Los resultados obtenidos en el presente trabajo concuerdan con los presentados por Vega-Cendejas et al. (1997) en la reserva de Celestún, donde mencionan que esta especie consume principalmente moluscos y crustáceos. Esto puede deberse a su aparato bucal grande y mandíbulas fuertes, que le permiten alimentarse de presas de gran tamaño, como los peces y los moluscos de la familia Marginellidae. Claro (1994) se refiere a esta especie como "oportunista" poblando los refugios artificiales ubicados sobre fondos de seibadal, donde los peces constituyen el $56.3 \%$ del espectro, mientras que los crustáceos sólo alcanzan el $8 \%$. El hábitat demersal y coloración críptica de esta especie, indica que es un predador que está al acecho de sus presas, ocultándose en las rocas o enterrándose en sustratos arenosos y de conchas. Su coloración obscura le permite no ser visible para muchas de sus presas. Por otra parte, Schmitter-Soto (1998) menciona que es una especie solitaria, que se alimenta de moluscos y crustáceos. Collette (2001) describe al pez sapo como voraces depredadores, de alimentación carnívora principalmente de peces, moluscos y crustáceos. Otro factor que podría ser importante en la variación de sus hábitos alimenticios son los cambios ambientales, al respecto Santamaría-Miranda et al. (2005) mencionan que a la variación en el consumo e intensidad de la alimentación de los peces están relacionadas con los cambios ambientales y las alteraciones que estos provocan en los alimentos. En la laguna de Chelem durante el periodo de estudio existe un mayor desarrollo de macrófitas, debido al incremento en las concentraciones de oxígeno por la alta incidencia lumínica que favorece su desarrollo (González 1995), sobre el cual habitan gran cantidad de copépodos, anfípodos y otros microcrustáceos.

En la composición de la dieta se determinó una variación ontogenética, en donde se presenta una tendencia con el crecimiento hacia la especialización en el consumo de peces. Los juveniles $(<6 \mathrm{~cm}$ de LE, Newman et al. 2004) ingieren principalmente microcrustáceos; mientras que, en ejemplares adultos el consumo de peces se incrementó hasta en un $96 \%$ del peso total del contenido alimenticio. Al respecto Claro (1994) menciona que el aumento de talla de los depredadores incrementa su capacidad para consumir presas mayores y aumentan, asimismo, sus posibilidades de selección. En relación a las diferencias tróficas ontogénicas, se ha observado que reflejan las capacidades de los peces de ciertas tallas para capturar alguna presa (Sumton y Greenwood 1990). Por tanto, a pesar de que el patrón de alimentación de los ejemplares juveniles basado en microcrustáceos (fuente rica en proteínas y con alto contenido energético) podría estar en función de los requerimientos energéticos de acuerdo al crecimiento (Deudero 2001). Por otro lado el consumo de microcrustáceos en las etapas tempranas del desarrollo de esta especie puede deberse también a su disponibilidad en el medio. Por consiguiente, estas variaciones durante la ontogenia se realizan siempre sustituyendo los organismos de menor contenido energético, como los invertebrados, por otros de mayor, como los peces. Otro aspecto importante de estos cambios ontogénicos en la dieta es que permiten una repartición de los recursos con otras especies, generando de esta forma mayores tasas de crecimiento entre ellas y reduciendo la competencia ínterespecífica (García- Berthou y Moreno-Amich 2000). La gran amplitud de su boca, como menciona Bohlke y Chaplin (1993) está en relación con 
su etapa de crecimiento y preferencias alimenticias. Esto corresponde con los resultados obtenidos sobre las preferencias alimenticias de ejemplares de $O$. phobetron mayores de 11.5 $\mathrm{cm}$ de LE los cuales consumen preferentemente peces y gasterópodos, mientras que individuos de menor talla, el principal recurso alimenticio lo constituyen los microcrustáceos. Estas variaciones constituyen adaptaciones evolutivas que permiten un mejor aprovechamiento en la cual se basa su alimentación (Claro 1994).

\section{AGRADECIMIENTOS}

Un agradecimiento especial a Mirella Hernández de Santillana por la ayuda proporcionada en la obtención de muestras y en la identificación de los contenidos estomacales, así como al grupo de trabajo que apoyo en las actividades de campo y laboratorio. Nuestro agradecimiento a los revisores anónimos cuyos comentarios y sugerencias enriquecieron el presente documento.

\section{RESUMEN}

El objetivo del presente estudio es evaluar la distribución y la preferencia alimenticia del pez sapo Opsanus phobetron en la Laguna de Chelem, Yucatán, México. Se recolectaron los peces de marzo 2002-enero 2003 en ocho estaciones distribuidas aleatoriamente. Los componentes del espectro trófico fueron analizados por medio del porcentaje de la abundancia relativa y la frecuencia de presencia. La similitud trófica entre las etapas ontogenéticas fue determinada usando el índice de Morisita (1959). Se capturó un total de 221 organismos, con una densidad y una biomasa de 92.09 ind $/ 100 \mathrm{~m}^{2}$ y de $930.39 \mathrm{~g} / 100 \mathrm{~m}^{2}$ respectivamente. Una misma estación registró los valores más altos de densidad y biomasa. El análisis de 94 de contenidos estomacales indicó una amplia generalización trófica, incluyendo 40 artículos alimenticios, y una gran variación ontogenética: las etapas juveniles consumen preferencialmente microcrustáceos, mientras que en los adultos el consumo de peces alcanza $96 \%$ de todo el contenido.

Palabras clave: Opsanus phobetron, distribución, hábitos alimenticios, Laguna de Chelem, México.

\section{REFERENCIAS}

Arceo, C. 2005. Ensamblajes de los peces de la reserva Bocas de Dzilam, Yucatán y su relación con variables Hidrológicas. Tesis de Maestría, CINVESTAV-IPN, Unidad Mérida, Yucatán, México.

Castro-Aguirre, J.L., P.H. Espinoza \& S.J. Schmitter. 1999. Ictiofauna Estuarino-Lagunar y Vicaria de México. Limusa, México D.F., México.

Claro, R. 1994. Ecología de los peces marinos de Cuba. Centro de Investigación de Quintana Roo. Editorial Academia, México D.F., México.

Collette, B.B. 2001.Opsanus dichrostomus ,a new toadfish (Teleostei: Batrachoididae ) from the Western Caribbean Sea and western Gulf of Mexico. Occasional Papers, Museum of Zoology, University of Michigan, Michigan, EEUU.

Bohlke, J. \& G. Chaplin. 1993. Fishes of the Bahamas and adjacent tropical waterns. University of Texas, Texas, EEUU.

De la Lanza, E. \& M.C. Canceres. 1994. Lagunas costeras y el litoral mexicano. Universidad Autónoma de Baja California Sur, México.

Deudero, S. 2001. Interspecific trophic relationship among pelagic fish species underneath FADs. J. Fish Biol. 58: 53-67.

Fischer, W. 1978. FAO Species Identification Sheets for Fishery Purposes. Western Central Atlantic, FAO, Roma, Italia.

García-Berthou, E. \& R. Moreno-Amich. 2000. Food of introduced pumpkinseed sunfish: ontogenetic diet shift and seasonal variation. J. Fish Biol. 57: 29-40.

Gonzáles, A. 1995. La comunidad de peces asociada al manglar de la laguna costera de Celestún, Yucatán, México. Tesis de Licenciatura, Univ. Nal. Autón. de México, México D.F., México.

Guitar, D.J. 1974. Sinopsis de los Peces Marinos de Cuba. Academia de Ciencias de Cuba. Instituto de Oceanología, La Habana, Cuba. Tomo IV.

Herrera-Silveira, J.A., J. Ramírez \& A. Zaldivar. 1998. Overview and characterization of the hydrology and primary producer communities of selected coastal lagoons of Yucatán, México. Aquatic Ecosystem Health and Management 1: 353-372. 
Horn, H.S. 1966. Measurement of overlap in comparative ecological studies. Am. Nat. 100: 419-424.

Hyslop, E.J. 1980. Stomach contents analysis a review of methods and their application. J. Fish. Biol. 17: 411-429.

Morisita, M. 1959. Measuring interespecific association and similarity between communities. Univ. Ser. Ed. (Biol.). 3: 65-80.

Newman, S.P., S.H. Gruber \& R.D. Handy. 2004. The scarecrow toadfish: habitat, abundance and size at maturity at Bimini, Bahamas. J. Fish Biol. 64: 248252.

Santamaría-Miranda, A., M. Saucedo-Lozano, M.N. Herrera-Moreno \& J.P. Apún-Molina. 2005. Hábitos alimenticios del pargo amarillo Lutjanus argentiventris y del pargo rojo Lutjanus colorado (Pises: Lutjanidae) en el norte de Sinaloa, México. Rev. Biol. Mar. Oceanog. 40: 33-44.

Schmitter-Soto, J.J. 1998. Catálogo de los peces continentales de Quintana Roo. ECOSUR, Chiapas, México.

Sumton, W. \& J. Greenwood. 1990. Pre-and post-flood feeding ecology of four species of juvenile fish from the Logan-Albert estuarine system, Moreton Bay, Queensland. Aust. J. Ma. Fresh. Res. 41: 795-806.

Tresierra, A.A. \& M.Z. Culquichicóm. 1993. Biología pesquera. Editorial Libertad, Trujillo, Perú.
Vargas, V.M. 2004. Composición y distribución íctica, durante la temporada de lluvias y nortes en el estero de Yucalpetén, Yucatán, México. Tesis de Licenciatura, Universidad Autónoma de Yucatán, México.

Vega-Cendejas, M.E. 1990. Interacción trófica entre dos bagres Arius melanopus (Agassiz, 1829) y Arius felis (Linnaeus, 1776) en las costas de Celestún Yucatán, México. An. Inst. Cienc. Del Mar y Limnol. Univ. Nal. Auton. México 15: 185-194.

Vega-Cendejas, M.E. 1998. Trama trófica de la comunidad nectónica asociada al ecosistema de manglar en el litoral Norte de Yucatán. Tesis de Doctorado, Fac. de Ciencias. Univ. Nal. Autón. de México, México.

Vega-Cendejas, M.E., M. Hernández de Santillana \& A.G. Cruz. 1997. Los peces de la reserva de Celestún. Pronatura Península de Yucatán, A.C. The Nature Conservancy. CINVESTAV-IPN. Unidad Mérida, Yucatán, México.

Walters, V. \& R. Robins. 1961. A new Toadfish (Batrachoididae) considered to be a Glacial Relict in the West Indies. Am. Mus. Nat. History 20: 467.

Wolda, H. 1981. Similarity indices, sample size and diversity. Oecología 50: 296-302.

Yáñez-Arancibia, A. 1976. Observaciones sobre Mugil curema valenciennes, en áreas naturales de crianza, alimentación, madurez, crecimiento y relaciones ecológicas. An. Inst. Centro. del Mar y Limnol. Univ. Nal. Autón. México 3: 92-125. 